\title{
High-repetition-rate femtosecond optical parametric chirped-pulse amplifier in the mid-infrared
}

\author{
C. Erny • L. Gallmann • U. Keller
}

Received: 3 February 2009 / Published online: 3 March 2009

(C) The Author(s) 2009. This article is published with open access at Springerlink.com

\begin{abstract}
We discuss a dual-stage optical parametric chirped-pulse amplifier generating sub-100-fs pulses in the mid-infrared at a repetition rate of $100 \mathrm{kHz}$. The system is based on a $1064 \mathrm{~nm}$ pump laser and a 3-4 $\mu \mathrm{m}$ difference frequency generation seed source derived from the output of a femtosecond fiber laser amplifier. Both lasers are commercially available, are diode-pumped, compact, and allow for turn-key operation. Here, we focus our discussion on the design and dimensioning of the optical parametric chirpedpulse amplifier. In particular, we review the available gain materials for mid-infrared generation and analyze the impact of different stretching scenarios. Timing jitter plays an important role in short-pulse parametric amplifier systems and is therefore studied in detail. The geometry of the amplifier stages is optimized through a full 3-dimensional simulation with the aim of maximizing gain bandwidth and output power. The optimized system yields output pulse energies exceeding $1 \mu \mathrm{J}$ and an overall gain larger than 50 $\mathrm{dB}$. The high repetition rate of the pump laser results in an unprecedented average power from a femtosecond parametric system at mid-infrared wavelengths. First experimental results confirm the design and the predictions of our theoretical model.
\end{abstract}

PACS 42.65.Yj · 42.65.Re · 42.60.By

C. Erny · L. Gallmann $(\bowtie) \cdot$ U. Keller

Institute of Quantum Electronics, Physics Department, ETH

Zurich, Wolfgang-Pauli Strasse 16, 8093 Zurich, Switzerland

e-mail: gallmann@phys.ethz.ch

Fax: +41-44-6331059

\section{Introduction}

Intense femtosecond mid-infrared laser sources in the wavelength range from 3 to $4 \mu \mathrm{m}$ are of interest for many applications, ranging from traditional ultrafast spectroscopy to high-field laser science. This wavelength range coincides with many important vibrational transitions in molecules [1-3]. In high-field science, mid-infrared laser sources are expected to significantly increase cut-off energies in highharmonic generation [4].

We discuss in this paper the design and dimensioning of a femtosecond optical parametric chirped-pulse amplifier (OPCPA, [5]) operating at high repetition rates in the mid-infrared. The OPCPA is based on a $1064 \mathrm{~nm}, 10 \mathrm{ps}$, $100 \mathrm{kHz}$ laser amplifier system as a pump and a $3-4 \mu \mathrm{m}$ seed source. The mid-infrared signal is obtained through difference frequency generation (DFG) from a $1.5 \mu \mathrm{m}$ femtosecond Er:fiber amplifier [6, 7]. Both laser systems are commercial products. These compact and diode-pumped lasers are reliable and enable turn-key operation.

No suitable laser gain medium exists for intense femtosecond pulse generation in the mid-infrared. Pulses in this regime are usually generated by nonlinear frequency conversion such as optical parametric generation (OPG) or optical parametric amplification (OPA). Recent developments in the field of diode-pumped solid-state and fiber lasers make it possible to advance to applications previously limited to conventional chirped pulse amplification (CPA, [8]) systems, while benefiting from compactness, efficiency, and reliability of this new laser technology. This recent progress enabled the demonstration of high-repetitionrate OPA sources in the visible to near-infrared based directly on diode-pumped laser technology [9-11]. A major benefit of parametric amplifiers is the relatively low thermal load in the gain material. This benefit can be exploited with 
Table 1 Previous femtosecond sources in the mid-infrared. The table is limited to sources with characterized output in the spectral range between 2.5 and $5 \mu \mathrm{m}$. All systems except the last two in this table have been pumped by Ti:sapphire lasers around $0.8 \mu \mathrm{m}$ wavelength

\begin{tabular}{|c|c|c|c|c|c|}
\hline Reference & $\begin{array}{l}\text { Repetition } \\
\text { rate }[\mathrm{kHz}]\end{array}$ & $\begin{array}{l}\text { Pulse } \\
\text { energy }[\mu \mathrm{J}]\end{array}$ & $\begin{array}{l}\text { Pulse } \\
\text { duration [fs] }\end{array}$ & $\begin{array}{l}\text { Output } \\
\text { center } \\
\text { wavelength }[\mu \mathrm{m}]\end{array}$ & Comments \\
\hline Brida et al. [29] & 1 & 2 & $\sim 30$ & $3.1-4.7$ & $\begin{array}{l}\text { Pulse duration not measured, transform limit } \\
\text { indicated instead }\end{array}$ \\
\hline Ghotbi et al. [12] & 1 & $\sim 15$ & 110 & 3.1 & Higher pulse energies below $2.5 \mu \mathrm{m}$ \\
\hline Fecko et al. [13] & 1 & $3-4$ & 45 & 3 & Pulse energy before compression \\
\hline Wang et al. [14] & 1 & 70 & $\sim 150$ & $2.9-4$ & \\
\hline Maekawa et al. [15] & 1 & $0.5-5$ & 55 & $3-8$ & Pulse duration measured at $3 \mu \mathrm{m}$ only \\
\hline Witte et al. [16] & 1 & $<1$ & $>190$ & $3-10$ & Maximum energy, shortest pulses at $3 \mu \mathrm{m}$ \\
\hline Kaindl et al. [17] & 1 & $<1$ & $50-150$ & $3-20$ & High energy, short pulses on short wavelength side \\
\hline Rotermund et al. [18] & 1 & 15 & 160 & 3.5 & \\
\hline Gale et al. [19] & 1 & $11-13$ & 200 & $2.6-3.6$ & \\
\hline Emmerichs et al. [20] & 1 & $6-16$ & $150-400$ & $2.8-3.4$ & \\
\hline Petrov et al. [21] & 1 & $0.5-7$ & $<200$ & $3-4.4$ & High energy on long wavelength side \\
\hline Seifert et al. [22] & 1 & $\sim 0.05$ & 160 & $3.3-10$ & Pulse duration measured at $4 \mu \mathrm{m}$ \\
\hline Golubovic et al. [23] & $100-150$ & $<0.016$ & $<50$ & $2.4-22$ & $\begin{array}{l}\text { Pulse duration estimated from signal-idler } \\
\text { crosscorrelation }\end{array}$ \\
\hline Gruetzmacher et al. [24] & 4 & $?$ & $<65$ & $2.7-4.7$ & Pulse energy unknown \\
\hline Holtom et al. [25] & 250 & 0.55 & 160 & $2.5-2.9$ & Energy and duration for $2.75 \mu \mathrm{m}$ \\
\hline Emmerichs et al. [26] & $7.6 \times 10^{4}$ & $10^{-4}$ & $200-400$ & $2.6-4$ & \\
\hline Laenen et al. [30] & 0.05 & $<2$ & $500-2600$ & $2.6-7$ & Pumped at $0.523 \mu \mathrm{m}$ and $1.047 \mu \mathrm{m}$ \\
\hline Erny et al. [34] & 100 & $\sim 1$ & 92 & $3.5,3.7$ & Pumped at $1.064 \mu \mathrm{m}$ \\
\hline
\end{tabular}

high-repetition-rate pump sources for relatively easy scaling of average power and repetition rate.

While mid-infrared pulses have been generated in the idler wave of the system reported by Marangoni et al. [11], their main interest was on the near-infrared output and the idler was therefore not further characterized. Currently, most femtosecond mid-infrared OPAs employ $800 \mathrm{~nm}$ Ti:sapphire pump sources [12-26]. This is the case for the commercial TOPAS system by Light Conversion Ltd./Coherent Inc. or the more recent results in the wavelength range around $2.1 \mu \mathrm{m}[27,28]$ or 3-4 $\mu \mathrm{m}$ [29]. The mJ-class CPA Ti:sapphire systems used in these setups represent a proven and well-established technology, but they are expensive and the overall set-up is rather large. In contrast to the diodepumped laser based OPAs, most of these systems operate at moderate repetition rates of $1-10 \mathrm{kHz}$. An early result by Laenen et al. [30] used 0.523 and $1.047 \mu \mathrm{m}$ pump wavelengths for the generation of femtosecond pulses in the 2.6 to $7 \mu \mathrm{m}$ wavelength interval, but their pump laser was flashlamp-pumped and thus limited to a repetition rate of $50 \mathrm{~Hz}$. Table 1 gives an overview of previous demonstrations of femtosecond pulses in the wavelength range from 2.5 to $5 \mu \mathrm{m}$. The same data are displayed in Fig. 1. This graph clearly demonstrates that the average output power of

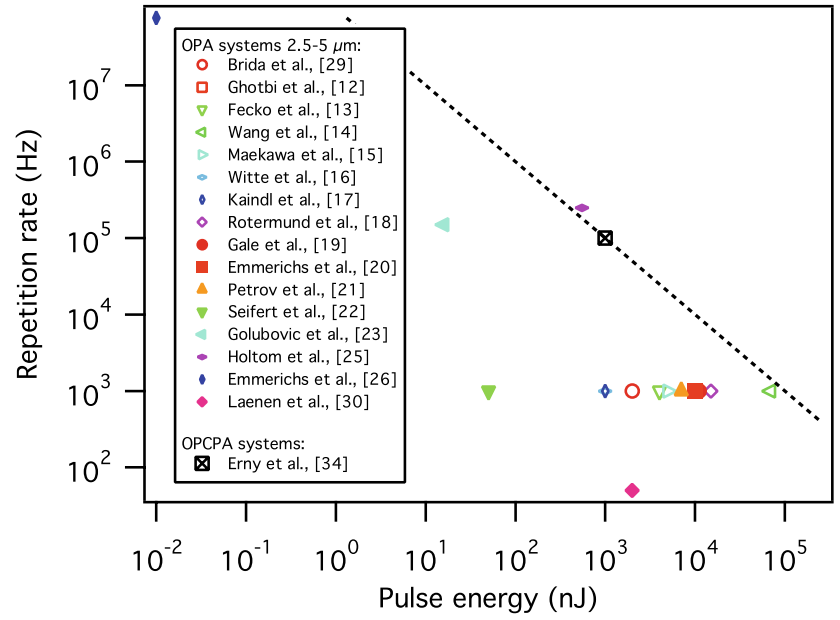

Fig. 1 Pulse energy and repetition rate of previous mid-infrared sources in the $2.5-5 \mu \mathrm{m}$ wavelength range compared to our OPCPA. Further details are given in Table 1 . The dashed line represents points of constant $100 \mathrm{~mW}$ average power

our system compares very well with the Ti:sapphire based approaches.

The high-repetition-rate OPCPA system which is discussed in this paper employs a tunable femtosecond mid- 
infrared source for seed generation [7]. It is based on difference frequency mixing of one output from a twobranch femtosecond Er:fiber amplifier [31] with the spectrally shifted output from the second branch. The spectral shift is generated in a highly nonlinear fiber (HNLF, [6]). The pump source in our experiments and simulations is the Duetto system from Time-Bandwidth-Products, which covers repetition rates in the range from $50 \mathrm{kHz}$ to $8 \mathrm{MHz}$ and pulse energies up to $200 \mu \mathrm{J}$ at a central wavelength of $1064 \mathrm{~nm}$. The 10 ps duration of its output pulses fits well to the attainable stretching factors of the seed. With simple and efficient bulk or prism stretchers we can easily stretch the mid-infrared seed to a duration on the order of several ps. For higher repetition rates in the $\mathrm{MHz}$ regime, the direct output from mode-locked thin-disk oscillators could be used as the pump source. Pulse energies of up to $26 \mu \mathrm{J}$ have been reported recently from such lasers [32, 33]. This is sufficient energy to operate the first amplification stage of the OPCPA discussed in this paper.

The OPCPA system design discussed in this paper was demonstrated experimentally [34] and yields results that are in excellent agreement with the data from the theoretical models presented here. Chalus et al. [35] recently reported a similar OPCPA design. Their design and modeling is however based on the assumption of a damage threshold of the nonlinear medium that is one order of magnitude higher than what we observe experimentally and assumed in the simulations presented here. This explains our choice of a two-stage amplification layout compared to the single-stage approach proposed by Chalus et al.

One of the challenges at mid-infrared wavelengths is posed by the choice of available optical materials for the nonlinear amplification and dispersive stretching of the pulse. Only a few materials are transparent between 3 and $4 \mu \mathrm{m}$. Most materials that are successfully used for $800 \mathrm{~nm}$ OPCPAs are no longer transparent at wavelengths longer than $2 \mu \mathrm{m}$. A prominent example for this is $\beta$-barium-borate (BBO). In the first part of this paper, we discuss the selection of nonlinear materials for a mid-infrared OPA together with their suitability for broadband amplification in this wavelength regime. Materials for quasi phase-matching (QPM) as well as for noncollinear birefringent phase matching are investigated.

In the second part, we present the design of a two-stage OPCPA, beginning with the optimization of the pulse duration ratio for pump and seed source. This is essential for a large amplification bandwidth and high conversion efficiency. For the same purpose we have studied the impact of the beam radii and have determined the optimal nonlinear crystal length. We show that for the available seed intensity it is necessary to distribute the amplification into two stages, a pre-amplification and a power-amplification stage, and we analyzed how the available pump power needs to be distributed between these two stages. The numerical results are supported by experimental data.

Numerical simulation makes it possible to look at pulse evolution inside the nonlinear crystal and get a detailed understanding of the optical parametric amplification process. We benefit from this capability when optimizing the crystal length. It turns out that the length of the nonlinear medium has to be chosen carefully. A crystal that is too short leads to spectral narrowing, while in a long medium back conversion from the signal to the pump can take place [36] or background noise from unsuppressed optical parametric generation can be amplified.

Our OPCPA system incorporates two independent modelocked laser oscillators-one seeding the fiber amplifier and one seeding the $1 \mu \mathrm{m}$ pump laser-that need to be synchronized to each other. In our case, this is done electronically with a phase-locked-loop (PLL) (model CLX-1100 from Time-Bandwidth Products). Due to the instantaneous nature of the optical parametric amplification process, the quality of this lock is crucial for the stability of the OPCPA system. Therefore, we have carefully analyzed the influence of the timing jitter between the two synchronized lasers onto the OPCPA system. These simulation results can be used to define the requirements on the tolerable jitter level of the PLL electronics.

In the last section, possible alternative schemes to increase the conversion efficiency and amplification bandwidth are discussed.

\section{Materials}

The nonlinear gain material is one of the key components for each OPA system, determining most of its properties. For high conversion efficiency the nonlinear material should ideally have a high damage threshold to apply tight focusing and a high nonlinear coefficient to keep the required crystal length short. A short crystal reduces the impact of the divergence mismatch between the involved beams. The dispersion relations should permit broadband phase-matching and the material should, of course, be transparent at all involved wavelengths. A list of suitable materials together with their basic optical properties can be found in Table 2 .

The table is divided into three sections. The first section shows the two materials that allow for quasi phase-matching (QPM): stoichiometric lithium tantalate $\left(\mathrm{LiTaO}_{3}\right)$ and magnesium doped lithium niobate $\left(\mathrm{MgO}: \mathrm{LiNbO}_{3}\right)$. Lithium niobate can also be used for birefringent phase-matching together with lithium iodate $\left(\mathrm{LiIO}_{3}\right)$ and potassium niobate $\left(\mathrm{KNbO}_{3}\right)$ as listed in the second section of Table 2. For comparison of the values with material properties available for a visible OPA, we listed $\beta$-barium-borate (BBO) in the last 
section of the table. The corresponding values for the visible OPA are calculated for $400 \mathrm{~nm}$ pump and a signal wavelength of $630 \mathrm{~nm}$.

The lithium based materials are all transparent up to at least $4800 \mathrm{~nm}\left(\mathrm{LiNbO}_{3}\right)$ [37]. This is sufficient for covering the tuning range of our DFG seed source, which is tunable between $3.2 \mu \mathrm{m}$ and $4.8 \mu \mathrm{m}$ [7]. The transparency of $\mathrm{LiNbO}_{3}$ is slightly decreasing for wavelength longer than $4 \mu \mathrm{m}$. In $\mathrm{KNbO}_{3}$, we observe an even more pronounced reduction in transparency beyond $4 \mu \mathrm{m}$.

A major benefit of quasi phase-matching is the ability of designing the nonlinear medium such that the largest nonlinear coefficient can be exploited for the nonlinear interaction, only reduced by the QPM-specific factor of $\pi / 2$. This leads to an effective nonlinear coefficient that is a factor 3 to 10 larger than attainable with birefringent phase-matching. The coupling coefficient $\kappa^{2}=\frac{d_{\text {eff }}^{2}}{n_{1} n_{2} n_{3} \lambda_{1} \lambda_{2} \lambda_{3}}$ for the three-wave optical parametric mixing process scales accordingly [38].

Table 2 also reveals that the coupling coefficient for QPM $\mathrm{LiNbO}_{3}$ is more than an order of magnitude higher than for bulk $\mathrm{LiIO}_{3} \cdot \mathrm{A} \mathrm{LiIO}_{3}$ crystal would thus have to be more than an order of magnitude longer than the periodically poled $\mathrm{LiNbO}_{3}$ crystal to achieve the same optical gain. However, noncollinear phase-matching, typically required for broadband amplification in birefringently phase-matched media, and Poynting vector walk-off limit the interaction length in the OPA crystal. In contrast, the QPM crystals can be operated in a collinear configuration, which results in a relatively high gain in a simple beam geometry and with short crystals.

For the two QPM materials, the coupling coefficient of $\mathrm{LiNbO}_{3}$ is a factor 2 larger than for $\mathrm{LiTaO}_{3}$, but the damage threshold of lithium tantalate is higher than for lithium niobate because of reduced photorefractive damage caused by green-induced infrared absorption [39, 40]. The gain bandwidth for the two materials is similar but limited. A larger gain bandwidth could be achieved by pumping the OPA at $800 \mathrm{~nm}$ (see Fig. 2) with a Ti:sapphire pump laser for ex-

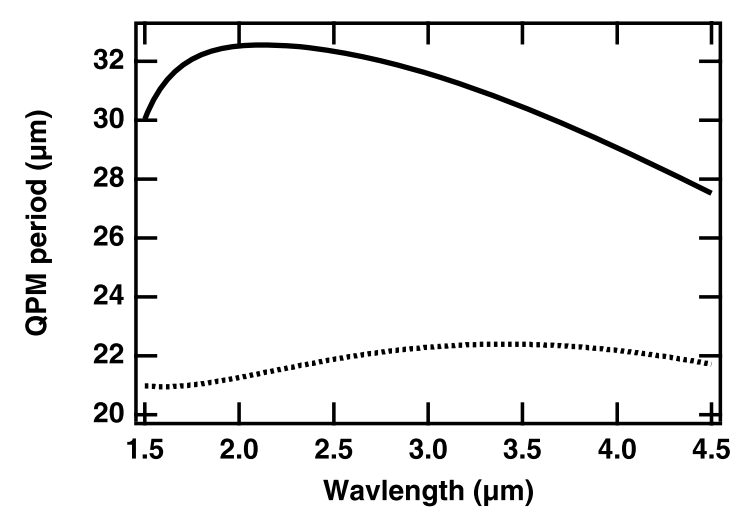

Fig. 2 Wavelength dispersion of the quasi phase-matching period for congruent periodically poled $\mathrm{LiNbO}_{3}$ with the pump at $1064 \mathrm{~nm}$ (solid line) and $800 \mathrm{~nm}$ (dashed line), respectively

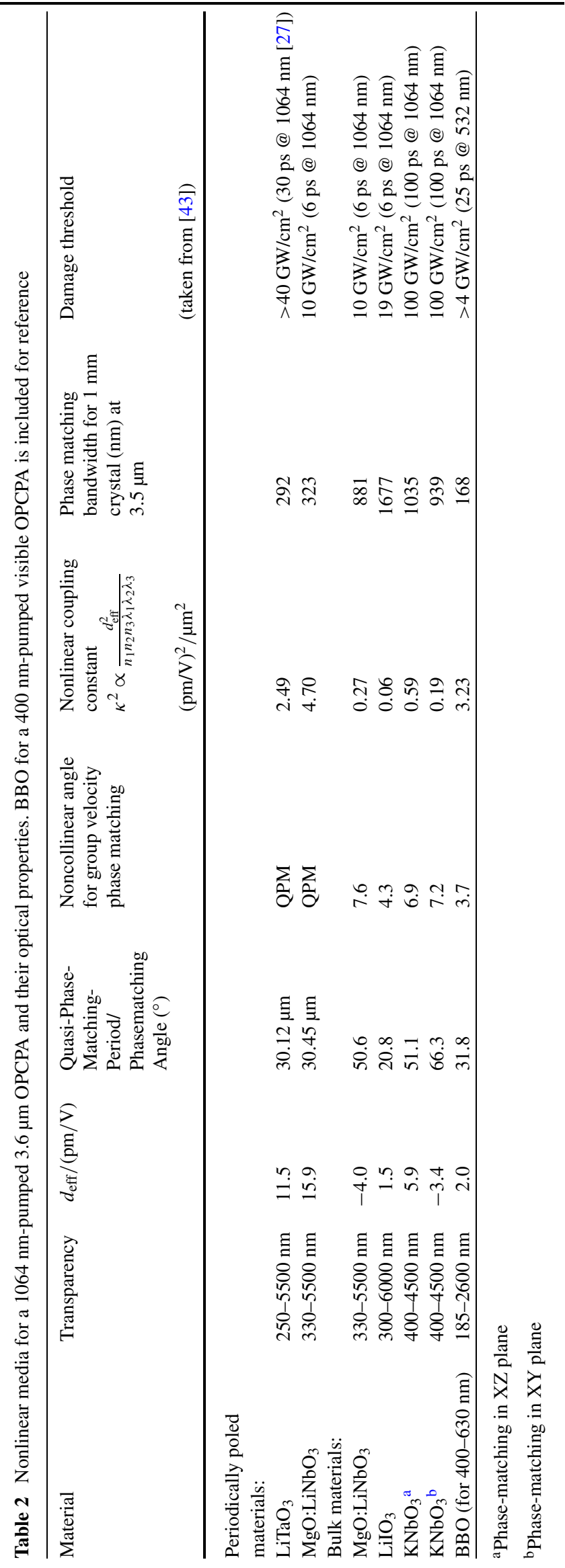


ample. However, this would come with all the drawbacks of Ti:sapphire laser systems such as lower repetition rate and efficiency while being larger in size and higher in cost and complexity. With a pump wavelength of $1 \mu \mathrm{m}$, the degeneracy of PPLN, which would allow for broadband amplification, is located at $2 \mu \mathrm{m}$ [41]. The downside of such a degenerate configuration is that it cannot be operated in a collinear geometry since the signal and idler are at the same wavelength.

The phase-matching bandwidth for an OPA is in first order inversely proportional to the group velocity mismatch $\mathrm{GVM}=1 / v_{\text {signal }}-1 / v_{\text {idler }}$. Broadband amplification can therefore be achieved when the group velocity of the signal and idler are identical. This condition can be reached by a noncollinear beam geometry [42]. Under the conditions of this configuration, an amplification bandwidth of up to $1.6 \mu \mathrm{m}$ is feasible with $\mathrm{LiIO}_{3}$ at $3.5 \mu \mathrm{m}$ center wavelength and assuming a $1 \mathrm{~mm}$ thick crystal (Table 1). This configuration comes, however, with the lowest available nonlinear coupling constant. The second largest amplification bandwidth is obtained with $\mathrm{KNbO}_{3}$ but compared to QPM $\mathrm{LiNbO}_{3}$, the coupling constant is still a factor 8 smaller.

Not only the effective nonlinearity but also the choice of interacting wavelengths plays an important role for a large nonlinear coupling coefficient. This is illustrated by comparing the values calculated for our mid-infrared OPA with those of a visible OPA based on BBO. While the effective nonlinear coefficient of $\mathrm{BBO}$ is among the lowest available in the list presented in Table 2, the shorter interacting wavelengths, with the pump at $400 \mathrm{~nm}$, the seed at $630 \mathrm{~nm}$, and the idler at $1100 \mathrm{~nm}$, lead to a coupling constant that is comparable to the one of $\mathrm{MgO}: \mathrm{LiNbO}_{3}$ for a $3.5 \mu \mathrm{m}$ OPA. Therefore the visible and the mid-infrared OPA show similar optical gain for comparable crystal lengths.

Many other nonlinear materials exist that are commonly used for mid-infrared nonlinear frequency mixing but are not listed in Table 2. Examples are mercury-thiogallate $\left(\mathrm{HgGa}_{2} \mathrm{~S}_{4}\right), \quad d_{\mathrm{eff}}=30.6 \mathrm{pm} / \mathrm{V}, \quad$ or silver-thiogallate $\left(\mathrm{AgGaS}_{2}\right), d_{\mathrm{eff}}=12.3 \mathrm{pm} / \mathrm{V}$ that both provide a high nonlinear coefficient and have recently become commercially available [43]. But compared to the materials mentioned above, they are either not completely transparent in the respective wavelength range, or, more important, they cannot provide broadband phase-matching.

Another promising material is GaAs, which shows a high nonlinear coefficient of $95 \mathrm{pm} / \mathrm{V}$ [44] in the case of quasi phase-matching. However, this material cannot be fabricated in the dimensions required by our OPCPA.

From the above discussion, we find that periodically poled $\mathrm{MgO}: \mathrm{LiNbO}_{3}$ is the most promising material for an OPA operated with our pump and seed sources. It allows for a high gain at relatively short crystal lengths. This is necessary to permit tight focusing to achieve the required high intensities.

\section{Simulation}

All simulations in this paper have been performed with a nonlinear propagation code by Arisholm [45]. The same code has been used successfully to model an $800 \mathrm{~nm}$ OPCPA laser system $[46,47]$. The program numerically solves the equations for second-order nonlinear frequency mixing for a full three-dimensional beam in an arbitrary birefringent crystal and takes into account the effects of depletion, diffraction, and walk-off. The software offers two numerical solvers: a Fourier-space method and a split-step method. For our simulations, we used the Fourier-space solver.

Where not stated differently, the following numerical values were used in our simulations. For the first amplification stage model we have assumed a $3.9 \mu \mathrm{J}, 1064 \mathrm{~nm}$, transform limited Gaussian pulse of $10 \mathrm{ps}$ duration as the pump pulse. The seed energy is $6.5 \mathrm{pJ}$, which corresponds to $0.5 \mathrm{~mW}$ of average mid-IR power at the $82 \mathrm{MHz}$ repetition rate of our seed source. The pump and the seed beams are focused to $49 \mu \mathrm{m}$ and $42 \mu \mathrm{m}$, respectively, corresponding to our experimental conditions. To get an estimate for the temporal input pulse shape we used the measured seed spectrum from our previous DFG experiment [7], centered at $3.6 \mu \mathrm{m}$ and with a full-width-at-half-maximum (FWHM) bandwidth of $326 \mathrm{~nm}$ (Fig. 3). The temporal pulse profile was calculated from this spectrum assuming a constant spectral phase, and it resulted in a pulse duration of $55 \mathrm{fs}$. From earlier simulations of our DFG seed source we know that the DFG output can safely be assumed to be close to transform limit. The two beams were overlapped temporally at the input of the crystal with the focus placed in the center of the crystal. The first amplification stage consists of a $3 \mathrm{~mm}$ long PPLN with an effective nonlinear coefficient of $d_{\text {eff }}=20 \mathrm{pm} / \mathrm{V}$. We have treated the nonlinear frequency mixing in the bulk approximation.

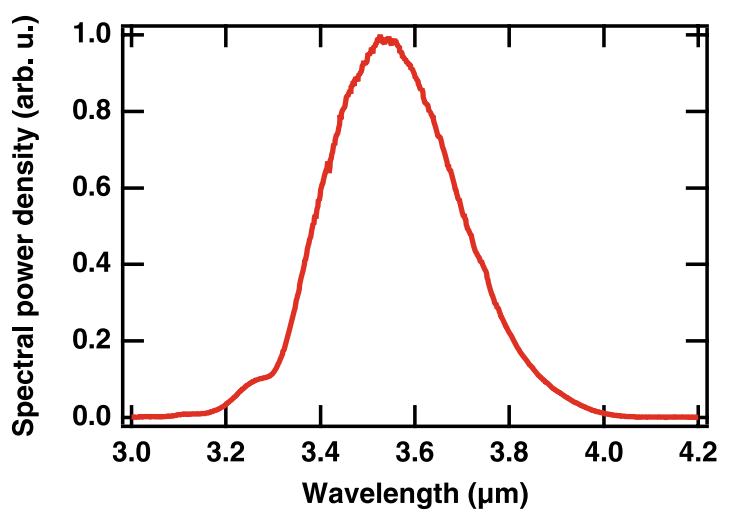

Fig. 3 Measured seed spectrum from our DFG setup. This spectrum is used as the input for the OPCPA simulations 


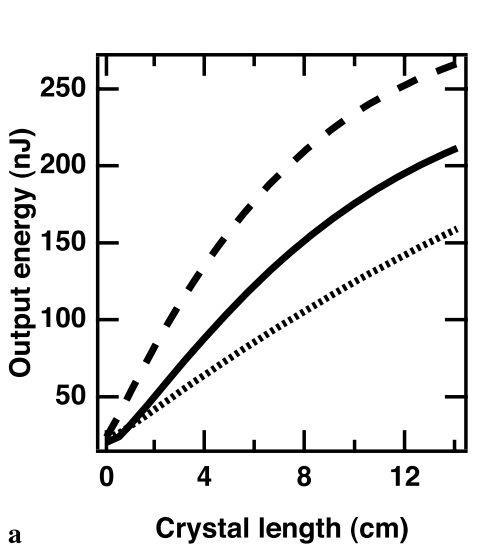

Fig. 4 Simulations of the first OPCPA stage consisting of a $3 \mathrm{~mm}$ PPLN crystal for various amounts of stretcher material (sapphire, solid line; germanium, dashed line; silicon, dotted line). (a) Achievable output power as a function of stretcher

\section{Stretching}

One of the key components of an OPCPA laser system is the stretching of the seed pulse. The highest gain can be expected when the pulse duration of the pump pulse and the seed pulse match. We show in this section that the ratio between the seed and the signal pulse has to be selected carefully to optimize the gain bandwidth. The low seed power of $<1 \mathrm{~mW}$ [7] together with the general experimental challenge to detect signals in this spectral range make prism or grating sequences difficult to align. We therefore limit our simulation to the case of stretching by bulk material.

There is only a small selection of sufficiently transparent dispersive materials available in the mid-infrared spectral region. The pulse-stretching factor was optimized with simulations for bulk sapphire $\left(\mathrm{Al}_{2} \mathrm{O}_{3}\right.$, ordinary refractive index), silicon $(\mathrm{Si})$, or germanium $(\mathrm{Ge})$ with a variable length between $1 \mathrm{~mm}$ and $140 \mathrm{~mm}$. While sapphire exhibits a negative group delay dispersion (GDD) of $-966 \mathrm{fs}^{2} / \mathrm{mm}$ at $3.5 \mu \mathrm{m}$, the semiconductor materials provide a positive GDD of $420 \mathrm{fs}^{2} / \mathrm{mm}$ and $1727 \mathrm{fs}^{2} / \mathrm{mm}$ for Si and Ge, respectively. This corresponds to stretched seed pulse durations of up to $8 \mathrm{ps}$ for the maximum material length considered. Sapphire and one of the semiconductors could be combined as a bulk stretcher/compressor pair, as they have opposite sign in GDD. However, their third-order dispersion (TOD) has the same sign and would therefore remain uncompensated, rendering this approach unsuitable for broadband pulse compression.

In our simulations, the seed and pump beams were temporarily overlapped at the end of the crystal, such that the pump depletion is only taking place in the central, most intense part of the pump pulse. The assumed pump intensity amounts to $7 \mathrm{GW} / \mathrm{cm}^{2}$, and the seed intensity ranges from

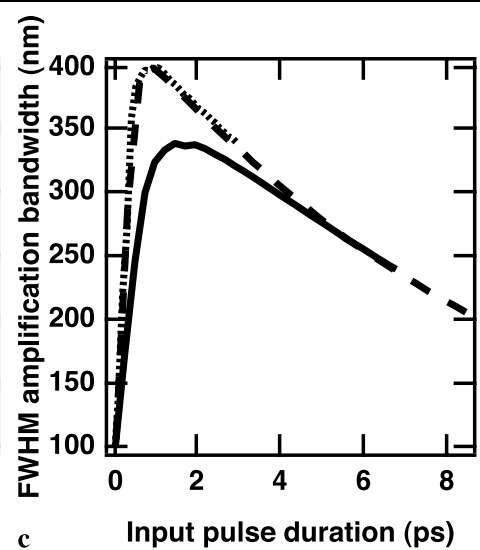

material length. (b) Full width at half maximum (FWHM) output bandwidth obtained for varying lengths of the three stretcher materials. (c) Gain bandwidth versus seed pulse duration

$2 \mathrm{~kW} / \mathrm{cm}^{2}$ and $2 \mathrm{MW} / \mathrm{cm}^{2}$, depending on the stretching factor. The pump intensity is set to our experimentally determined damage threshold for the nonlinear medium.

The amplified output power shows the same qualitative behavior for all three materials (Fig. 4a). With increasing stretcher material length and seed pulse duration the output energy rises linearly and then starts to saturate. For short input pulses, the rising slopes of the three curves are correlated to the GDD of the applied materials.

The gain bandwidth shows a strong dependency on the ratio between pump and seed pulse duration (Fig. $4 \mathrm{~b}$ and c). For short pulses, below 0.5 ps and 1.5 ps for the semiconductors and sapphire, respectively, the OPA shows a strong spectral gain narrowing, which is reduced as the input pulse becomes longer. The bandwidth reaches a maximum of $390 \mathrm{~nm}$ for a $1 \mathrm{ps}$ pulse stretched by germanium or silicon. The maximum bandwidth of $338 \mathrm{~nm}$ is obtained when stretching the pulse to a duration of $1.5 \mathrm{ps}$ with sapphire. Further stretching leads to more output power but a reduced gain bandwidth. The observed behavior is a result of the interaction between the chirp of the seed pulses and gain saturation.

Our observations can be explained as follows. Three regimes exist for the ratio of the seed and the pump pulse durations. In the first regime, the seed pulse is close to its transform limit and therefore much shorter than the pump pulse. The seed experiences nearly constant pump amplitude over its entire duration such that, even if gain saturation is taken into account for a nearly transform limited seed pulse, all spectral components are exposed to the same pump intensity. The gain bandwidth is therefore only limited by the phase-matching bandwidth of the nonlinear crystal. For a $3 \mathrm{~mm}$ long PPLN this is approximately $100 \mathrm{~nm}$.

In the second regime, the stretched seed pulse is still considered short compared to the pump pulse, but cannot 
be treated transform limited anymore. For such a stretched seed pulse, the spectral wings map to its temporal wings. When the OPA is operated with strong pump saturation, the more intense spectral components of the seed can deplete the pump pulse earlier than the weaker components located in the spectral wings. By burning a temporal hole into the pump pulse, the central spectral components effectively reduce the available pump energy. The spectral wings do not temporally overlap with this hole and therefore have their own pump energy reservoir. Therefore they are further amplified by an unsaturated pump. This leads to a reduced spectral narrowing.

In the third regime, the stretched seed pulse becomes comparable to the pump pulse duration. The temporal pump pulse profile now needs to be taken into account. The central part of the seed pulse still overlaps with the same part of the pump pulse as in the previous regime and therefore experiences similar gain. The temporal and thus also the spectral wings of the seed pulse now overlap with the lower intensity temporal wings of the pump pulse. As a consequence the wings of the seed become less amplified than the central part. This obviously results in significant gain narrowing.

We aim to generate the shortest possible pulses. Therefore we want to operate the OPCPA with a stretching factor yielding maximum amplification bandwidth corresponding to the second regime discussed above. From the simulation results depicted in Fig. 4 we can extract the optimum amount of stretcher material. Due to its relatively high GDD per unit material length only $16 \mathrm{~mm}$ of germanium are required, which results in a bandwidth of $397 \mathrm{~nm}$ and an output pulse energy of $69 \mathrm{~nJ}$. A silicon material length of $51 \mathrm{~mm}$ yields the same bandwidth at a pulse energy of $75.8 \mathrm{~nJ}$. Stretching with negative GDD from $36 \mathrm{~mm}$ of sapphire leads to a bandwidth of $338 \mathrm{~nm}$ and an output energy of $80 \mathrm{~nJ}$. By slightly increasing the amount of sapphire to $51 \mathrm{~mm}$, it is possible to increase the pulse energy to $107 \mathrm{~nJ}$ with an acceptable reduction in bandwidth to $329 \mathrm{~nm}$. Significantly more silicon would be required for similar performance: $91 \mathrm{~mm}$ of silicon yields $116 \mathrm{~nJ}$ with a $370-\mathrm{nm}$ bandwidth.

These results demonstrate that the positive GDD from the semiconductor materials is capable of providing a slightly higher gain bandwidth than the negative GDD from sapphire. The main advantage of sapphire lies in the lower sensitivity of the OPA bandwidth to the stretcher length. Even a sapphire crystal that was chosen too long by $50 \%$ does not significantly reduce the gain bandwidth.

Germanium and silicon show nearly the same performance when comparing bandwidth and output power. The main difference between the two is the required stretcher length. The ratio of GDD to TOD is different for the two semiconductor materials, which gives a clear indication that GDD plays a more important role in the OPA process than the TOD. From a practical point of view, silicon has the advantage that it is transparent from the mid-infrared to a wavelength of $1100 \mathrm{~nm}$. It is therefore possible to use the idler output from our collinear DFG seed source located at $1500 \mathrm{~nm}$ as an alignment beam for the OPA.

Our optimization leads us to select the $51 \mathrm{~mm}$ sapphire as the optimal stretcher. As will be shown below, this choice is confirmed by our experiments.

\section{First amplification stage}

In the previous section, we based our simulations on a $3 \mathrm{~mm}$ PPLN and beam radii of $49 \mu \mathrm{m}$ for the pump and $42 \mu \mathrm{m}$ for the seed, corresponding to the values from our experiment. In this section, we discuss the optimization of the nonlinear medium length and the beam radii with the help of our simulation tool. Important for this optimization is the capability of our simulation code to give insight into the evolution of the amplification process along the crystal. Our results show that the previously selected values are close to the optimum.

The most important parameters of the first amplification stage are gain, conversion efficiency and amplification bandwidth. Gain should be high so that the number of amplification stages can be kept small. The conversion efficiency should be maximized to make best use of the available pump power. A fundamental upper limit to conversion efficiency comes from the mismatch of the seed and pump pulse durations, which is necessary for maximum amplification bandwidth as shown in the previous section.

The simulation of the first OPA stage was done for a range of $1 / e^{2}$ beam radii between $20 \mu \mathrm{m}$ and up to $180 \mu \mathrm{m}$. We were assuming identical beam sizes for pump and seed. For each individual beam radius we have performed an intensity scan by varying the pump energy until we reach the damage threshold of the OPA crystal. To obtain insight into evolution of the amplification along the nonlinear medium, the crystal was divided into thin slices with the calculated data recorded for each of these slices. This enables us to determine the optimum crystal length and is ultimately used to decide on the number of amplification stages needed for our OPCPA system.

The results of our simulations are shown in Fig. 5. Figure 5a shows the OPA mid-infrared output power as a function of pump power for different choices of the beam radii. An increase in pump beam radius needs to be compensated for by an increase in pump power to maintain the same pump intensity. Since our seed source, on the other hand, only provides a fixed output power, seed intensity drops with increasing seed beam radius. This affects the coupling strength between the pump and the seed field. Therefore the maximum possible conversion efficiency reached at the damage threshold of the nonlinear medium decreases with increasing beam radii whereas the achievable output energy rises (Fig. 5b). 

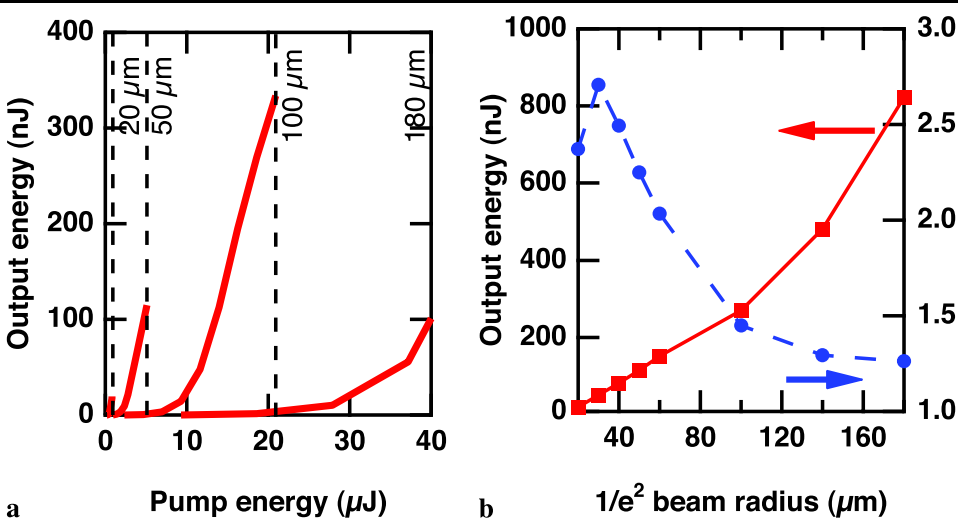

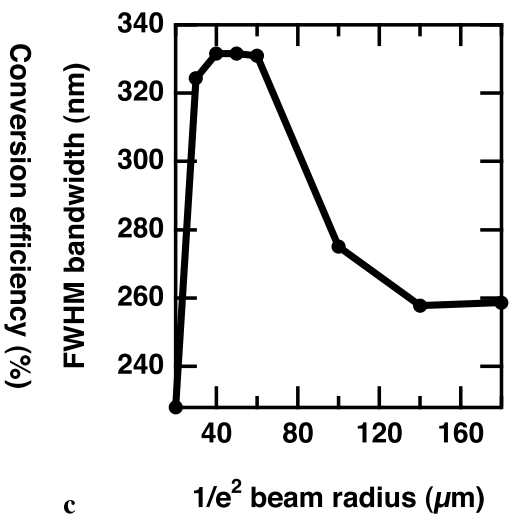

Fig. 5 Simulation results for the first OPA stage. (a) OPA output power versus pump power for different beam radii $(20 \mu \mathrm{m}, 50 \mu \mathrm{m}$, $100 \mu \mathrm{m}$, and $180 \mu \mathrm{m})$. The vertical lines mark the damage threshold for the different beam radii with the left-most line corresponding to the smallest radius and the right-most to the $100 \mu \mathrm{m}$ radius. The

For each beam radius the highest energy was always reached at the end of the $3 \mathrm{~mm}$ PPLN. The conversion efficiency shows a maximum of $2.7 \%$ at a beam radius of $30 \mu \mathrm{m}$ and drops to $2.4 \%$ towards $20 \mu \mathrm{m}$ and to $1.24 \%$ towards $180 \mu \mathrm{m}$. The decrease in coupling strength can be compensated for by increasing pump intensity, which is however limited by the damage threshold, or by choosing a longer crystal. The output energy at damage threshold amounts to $19 \mathrm{~nJ}$ for a $20 \mu \mathrm{m}$ beam radius when pumped with $700 \mathrm{~nJ}$ and rises up to $0.82 \mu \mathrm{J}$ for a radius of $180 \mu \mathrm{m}$ with a corresponding pump energy of $50 \mu \mathrm{J}$. The spectral bandwidth of the amplified pulse shows a maximum of $330 \mathrm{~nm}$ between 30 and $60 \mu \mathrm{m}$ radius and drops significantly towards smaller and larger radii (Fig. 5c). We therefore can conclude that our femtosecond OPA should be operated with a beam radius between $30 \mu \mathrm{m}$ and $60 \mu \mathrm{m}$ for maximum bandwidth and reasonable efficiency. We have selected a radius of $50 \mu \mathrm{m}$ as a compromise between efficiency and output energy and predict an output energy of $115 \mathrm{~nJ}$, a FWHM bandwidth of $331 \mathrm{~nm}$, and a conversion efficiency of $2.25 \%$. The corresponding spectrum supports $80 \mathrm{fs}$ pulses.

Through the calculation of the nonlinear interaction on a fine grid along the propagation axis, we are able to gain insight into the broadband, short pulse amplification process in a regime of high pump depletion. As expected, the energy of the mid-infrared pulses first increases exponentially, while saturation starts to become significant towards the end of the crystal (Fig. 6a). The bandwidth of the amplified signal exhibits a strong gain narrowing on propagation through the first half of the crystal. This can already be predicted within the undepleted plain-wave approximation. The bandwidth reaches a minimum of below $200 \mathrm{~nm}$ at $2.1 \mathrm{~mm}$ but starts to recover afterwards. An analogous behavior can be observed for the pulse duration of the stretched seed pulse. To understand the dynamics of this process, we have plotted damage threshold at $180 \mu \mathrm{m}$ is at $65 \mu \mathrm{J}$. (b) Maximum output energy achievable when reaching the damage threshold (red solid line) and the corresponding conversion efficiency (blue dashed line). (c) Full width at half maximum (FWHM) amplification bandwidth as a function of beam radius
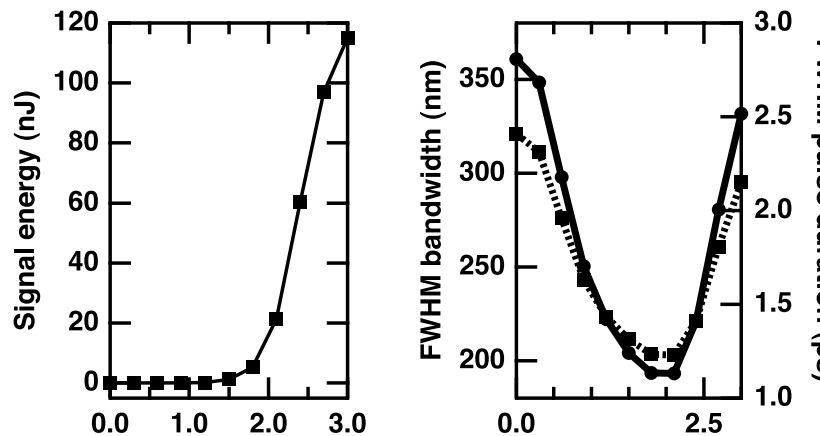

a Position inside PPLN (mm) b

Position inside PPLN (mm)

Fig. 6 Evolution of pulse energy, bandwidth and pulse duration along the $3 \mathrm{~mm}$ PPLN crystal of the first OPA stage. (a) Energy in the mid-infrared beam at different positions inside the crystal. (b) Mid-infrared full width at half maximum (FWHM) bandwidth (solid line) and pulse duration (dashed line) along propagation axis. A minimum is reached around $2 \mathrm{~mm}$ and the output values recover to close to the initial values

the temporal intensity profiles of the seed and pump pulse at six positions inside the crystal (Fig. 7). On the first $1.2 \mathrm{~mm}$ of the nonlinear interaction, a significant shortening of the seed pulse takes place, while the gain factor amounts to only 41 and no depletion of the pump pulse can be recognized. The depletion of the pump pulse starts to manifest itself after $1.8 \mathrm{~mm}$, where a gain of 785 is reached. This is the position where the pulse duration of the seed as well as its spectral bandwidth reaches the minimum (Fig. 6b). The slightly modulated pump pulse profile enhances the wings of the mid-infrared pulse compared to its central part. This effect becomes more pronounced the longer the two pulses interact (see Fig. 7 at $2.4 \mathrm{~mm}$ and $3.0 \mathrm{~mm}$ ). Towards $3.0 \mathrm{~mm}$, a strong temporal broadening can be observed. For the strongly linearly chirped mid-infrared pulse this is equiv- 

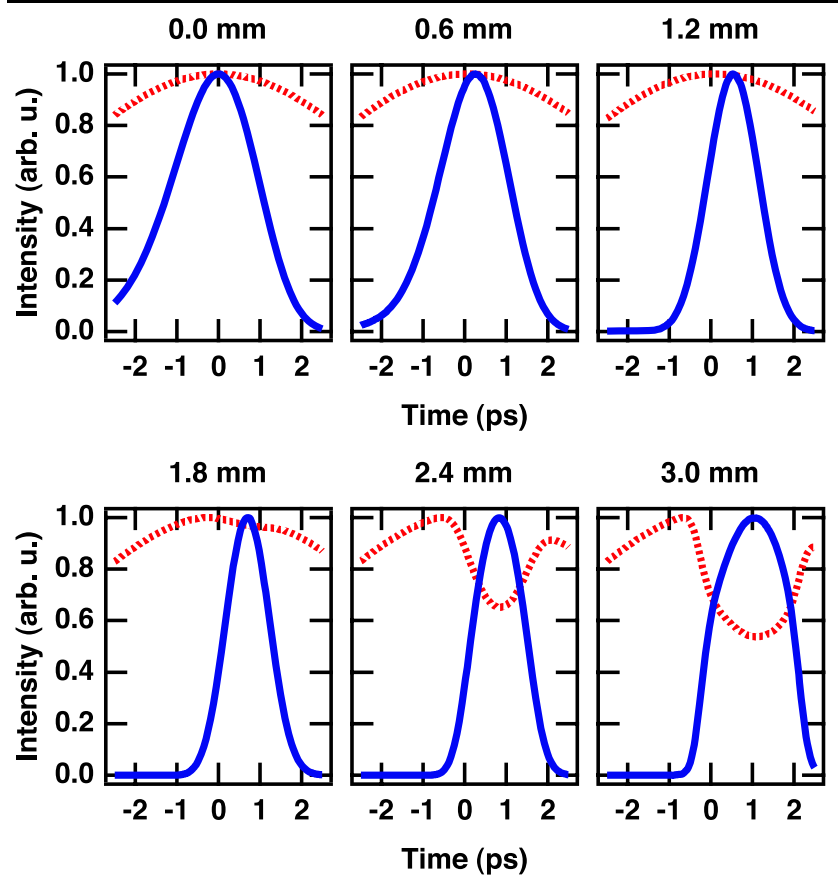

Fig. 7 Temporal profile of the pump pulse (red dotted line) and the signal pulse (blue solid line) at various positions along the crystal. No saturation effect is visible for a propagation length of up to $1.8 \mathrm{~mm}$. Between $1.8 \mathrm{~mm}$ and the end of our $3 \mathrm{~mm}$ crystal, the pump pulse becomes strongly depleted. Pump depletion leads to a temporal and spectral broadening of the seed. The pump energy is $4.3 \mu \mathrm{J}$ and the beam radii are $50 \mu \mathrm{m}$

alent to a strong spectral broadening (Fig. 6b). Therefore pump depletion can support both a high gain of 17000 and a broader spectral bandwidth. At the end of the crystal the spectral bandwidth has recovered from its minimum of below $200 \mathrm{~nm}$ back to $330 \mathrm{~nm}$. This is $90 \%$ of the initial input bandwidth of $360 \mathrm{~nm}$.

From our simulations we find that a shorter crystal would only reduce the conversion efficiency and lead to a smaller gain bandwidth. An even longer crystal has the benefit of even higher gain, but the crystal length is limited by the onset of significant optical parametric generation (OPG). OPG does not require a seed but starts directly from quantum fluctuations. Even though it can be expected that the OPG beam is initially much weaker than the amplified seed, the two processes will compete for gain. The OPG radiation has the competitive advantage compared to the desired seed amplification that it is always perfectly temporally and spatially overlapped with the pump. The amplification of the OPG signal may thus saturate later than the desired seed amplification process. Significant OPG contributions increase the noise level in the output beam. We simulate the OPG process by including quantum noise modeled by numerical white noise into our calculations. The simulated noise starts to build up and destabilizes our simulations if the nonlin-
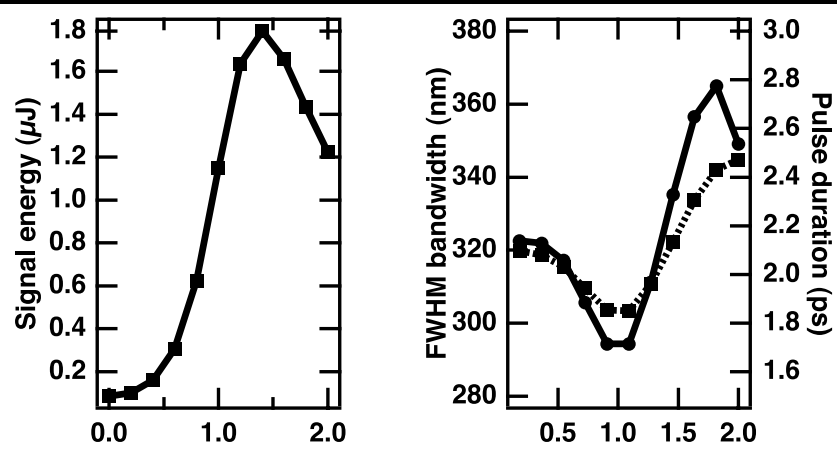

a Position inside PPLN (mm)

b Position inside PPLN (mm)

Fig. 8 Evolution of amplified mid-infrared power, bandwidth and pulse duration along the 2nd OPA crystal. The OPA was pumped at $50 \mu \mathrm{J}$, with a seed pulse energy of $84 \mathrm{~nJ}$ and beam radii of $180 \mu \mathrm{m}$. (a) The amplified energy shows a maximum at $1.4 \mathrm{~mm}$ crystal length. Afterwards back conversion from the mid-infrared back to $1.5 \mu \mathrm{m}$ and $1 \mu \mathrm{m}$ starts to become significant. (b) Spectral bandwidth (solid line) and pulse duration along the crystal length (dashed line)

ear propagation is extended well beyond the $3 \mathrm{~mm}$ crystal length.

Our simulations for the first amplification stage lead us to the following design: a $3 \mathrm{~mm}$ long MgO:PPLN with beam radii of around $50 \mu \mathrm{m}$ for the pump and the seed pulse. The pump pulse energy is set to $3.9 \mu \mathrm{J}$.

\section{Second amplification stage}

We have demonstrated in the previous section that a single OPA stage is not sufficient to fully benefit from the available $10-\mathrm{W}$ average pump power while maintaining efficient and broadband amplification. Therefore, we have repeated the same types of simulations performed for the first OPA stage in the context of a subsequent second OPA.

The simulated mid-infrared output from the first optimized amplification stage was used as the input for the second amplification stage. Taking into account the Fresnel losses at the uncoated surface of the PPLN, we chose an effective input energy of $84 \mathrm{~nJ}$ in the mid-IR. We first performed simulations at various positions along a $2 \mathrm{~mm}$ long PPLN crystal assuming beam radii of $180 \mu \mathrm{m}$ and a pump energy of $50 \mu \mathrm{J}$, close to the damage threshold of the crystal. With the much higher seed intensity compared to the first OPA, the pump pulse is depleted much earlier inside the crystal (Fig. 8a). The strong pump depletion may cause some back conversion for which sum-frequency generation is dominating the nonlinear interaction instead of OPA. As a consequence the energy flows back from the signal and the idler wave to the $1-\mu \mathrm{m}$ pump. This can significantly lower the achievable output power. As can be seen in Fig. $8 \mathrm{a}, 1.2 \mu \mathrm{J}$ of output energy is generated after $2 \mathrm{~mm}$ of PPLN whereas a maximum of $1.79 \mu \mathrm{J}$ is reached at $1.4 \mathrm{~mm}$ inside the material. The conversion efficiency is nearly $50 \%$ higher for 

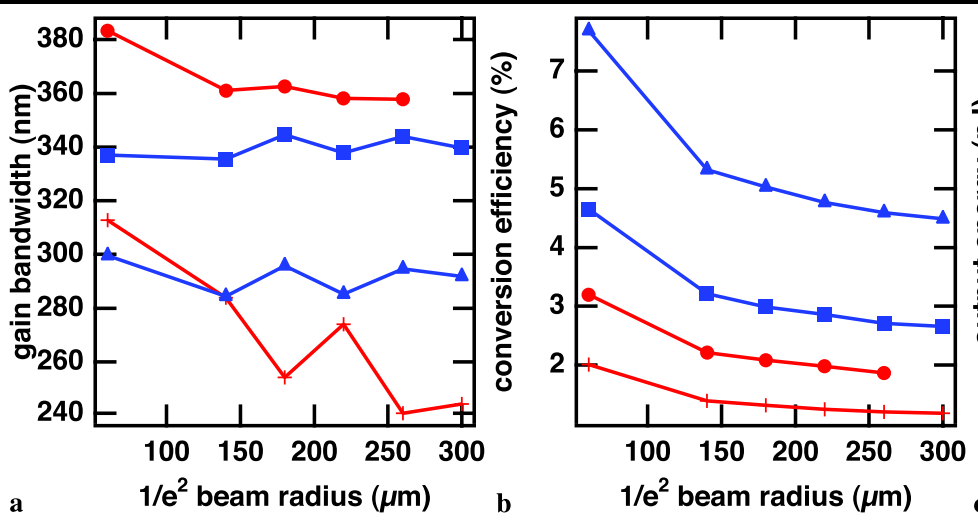

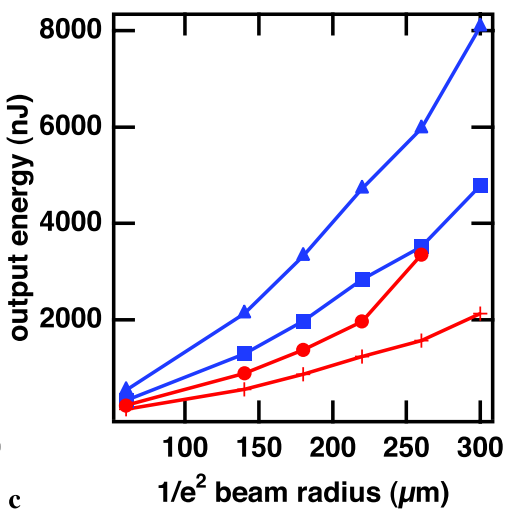

Fig. 9 Optimization of beam radii and stretching factor for the second OPA stage. The stretcher consisted of $100 \mathrm{~mm}$ (triangles) and $50 \mathrm{~mm}$ (squares) of $\mathrm{Al}_{2} \mathrm{O}_{3}$ and $50 \mathrm{~mm}$ (circles) and $25 \mathrm{~mm}$ (crosses) of Si. (a) Gain bandwidth for different pump intensities and stretchers. The highest bandwidth is obtained with
$50 \mathrm{~mm} \mathrm{Si}$, followed by $50 \mathrm{~mm} \mathrm{Al}_{2} \mathrm{O}_{3}$. (b) The highest conversion efficiency $P_{\text {out }} / P_{\text {Pump } 1064 \tilde{n} m}$ is achieved when stretching the pulses with $100 \mathrm{~mm} \mathrm{Al}_{2} \mathrm{O}_{3}$, followed by $50 \mathrm{~mm} \mathrm{Al}_{2} \mathrm{O}_{3}$. (c) Output energy obtained when pumping at the damage threshold

Table 3 Influence of the stretcher material choice on the output performance of the two OPA stages. The first OPA is pumped at $3.9 \mu \mathrm{J}$, whereas second OPA is pumped at $50 \mu \mathrm{J}$

\begin{tabular}{lllll}
\hline Stretcher material & $\begin{array}{l}\text { 1st OPA } \\
\text { output energy }(\mathrm{nJ})\end{array}$ & $\begin{array}{l}\text { 1st OPA FWHM } \\
\text { gain bandwidth }(\mathrm{nm})\end{array}$ & $\begin{array}{l}\text { 2nd OPA } \\
\text { output energy }(\mu \mathrm{J})\end{array}$ & $\begin{array}{l}\text { 2nd OPA FWHM } \\
\text { gain bandwidth }(\mathrm{nm})\end{array}$ \\
\hline $50 \mathrm{~mm} \mathrm{Al}_{2} \mathrm{O}_{3}$ & 115 & 332 & 1.79 & 335 \\
$100 \mathrm{~mm} \mathrm{Al}_{2} \mathrm{O}_{3}$ & 160 & 260 & 3 & 280 \\
$25 \mathrm{~mm} \mathrm{Si}$ & 43 & 354 & 0.77 & 250 \\
$50 \mathrm{~mm} \mathrm{Si}$ & 68 & 360 & 1.25 & 357 \\
\hline
\end{tabular}

the optimized length of PPLN. The spectral bandwidth and pulse duration show the same behavior as was seen with the first OPA (Fig. 8b). Spectral broadening due to pump depletion follows an initial spectral narrowing. The effect is much less pronounced than in the first OPA stage. Our simulation results clearly show that it is crucial to choose the crystal length sufficiently short to optimize the output power and conversion efficiency.

We ran an additional simulation with the goal to determine the optimum beam radii for the mid-infrared seed and the pump. The simulated radii covered the range from $60 \mu \mathrm{m}$ to $300 \mu \mathrm{m}$. The seed intensity therefore ranges from 10 to $300 \mathrm{MW} / \mathrm{cm}^{2}$. The pump energy was set to operate close to the damage threshold of the PPLN crystal. Pump energies of up to $100 \mu \mathrm{J}$ were used, corresponding to the maximum available energy from our $10-\mathrm{W}, 100 \mathrm{kHz}$ Duetto pump laser. To additionally investigate the influence of the stretcher material on the performance of the two-stage OPA we have repeated the same calculations for pulse stretchers made up from $50 \mathrm{~mm}$ and $100 \mathrm{~mm}$ of $\mathrm{Al}_{2} \mathrm{O}_{3}$ or $25 \mathrm{~mm}$ and $50 \mathrm{~mm}$ of Si. The results shown in Fig. 9 are read out after the OPA crystal length yielding the highest conversion efficiency. The detailed numerical results of our simulations can be found in Table 3. The comparison of the four possible pulse stretchers clearly shows that $50 \mathrm{~mm}$ of $\mathrm{Al}_{2} \mathrm{O}_{3}$ provides the best performance for our broadband OPCPA. It results in the highest available gain bandwidth at high conversion efficiency.

In contrast to the first OPA stage we did not observe a clear limit for power scaling. In the second OPA stage the seed intensity is sufficiently high to obtain power scaling over a wide range. A practical scaling limit of the second OPA is set by the available crystal sizes. The $180 \mu \mathrm{m}$ beam radius that we determined to be the optimum for our available pump energy is already large compared to the typical $1 \mathrm{~mm}$ clear apertures of commercial PPLNs. Larger clear apertures are, however, becoming commercially available. With larger apertures, bigger beam radii and higher pump energies could be used. Alternatively, one could exchange the PPLN by PPLT, which offers a higher damage threshold ( $>40 \mathrm{GW} / \mathrm{cm}^{2}$, for $30 \mathrm{ps}$ pulses at $1064 \mathrm{~nm} \mathrm{[27]).} \mathrm{The}$ higher damage threshold allows for increasing the pump energy to $330 \mu \mathrm{J}$ at the beam radius of $180 \mu \mathrm{m}$. With these parameters, the input beam is amplified to $11 \mu \mathrm{J}$ and the generated pulses possess a bandwidth of $390 \mathrm{~nm}$. This result corresponds to a conversion efficiency of $2.7 \%$.

We implemented the optimized OPCPA design in our laboratory [34]. Because we did not use fully optimized op- 
tics, the effective pump energy available in the second OPA was only $40 \mu \mathrm{J}$ instead of the $50 \mu \mathrm{J}$ used in the simulations. All other experimental parameters closely match the simulations. When we take the lower second-stage pump energy into account in our model, we find very good agreement between theory and experiment. We have measured a pulse energy of $100 \mathrm{~nJ}$ after the first amplification stage and $1 \mu \mathrm{J}$ after the second amplification stage with an average power of $100 \mathrm{~mW}$ at $100 \mathrm{kHz}$ repetition rate. Simulations predict the somewhat higher pulse energy of $1.37 \mu \mathrm{J}$. When operating the OPCPA at a center wavelength of $3.6 \mu \mathrm{m}$, we measured for the output pulses a FWHM bandwidth of $332 \mathrm{~nm}$ after the first OPA and $311 \mathrm{~nm}$ after the second OPA, which are close to our simulations predicting a bandwidth of $323 \mathrm{~nm}$ for the first and $313 \mathrm{~nm}$ for the second amplification stage. We characterized the compressed output pulses of the OPCPA using frequency-resolved optical gating and obtained a pulse duration of the recompressed OPCPA output of 95 fs. This corresponds to a 8-9 cycle pulse at this particular wavelength. We have not measured significant power from OPG. More experimental results from our system at center wavelengths of $3.5 \mu \mathrm{m}$ and $3.7 \mu \mathrm{m}$ can be found in [34].

\section{Timing jitter}

One of the major practical issues of a OPCPA system based on two independent short pulse laser systems is the repetition-rate locking of the two lasers with respect to each other. This is typically done by an electronic phase-locked loop (PLL) [48]. The stabilization is good as long as the individual lasers exhibit no significant noise in average output power and pulse repetition rates. Therefore a solid mechanical design and a good isolation from environmental influences are a prerequisite to minimize the residual timing jitter between the two lasers. We have simulated the influence of a residual timing jitter on the performance of our twostage OPCPA design. The two-stage amplification system has been modeled in two steps. The seed was identical to the previous simulation runs, stretched by $50 \mathrm{~mm} \mathrm{Al}_{2} \mathrm{O}_{3}$. Pump and seed were focused down to $49 \mu \mathrm{m}$ and $42 \mu \mathrm{m}$, respectively, inside the $3 \mathrm{~mm}$ PPLN of the first OPA. The output pulse of the first stage was then calculated for different time delays between seed and pump. For each of these time delays, the temporal profile of the output pulse is obtained by a weighted average across the transverse beam profile. The output from the first stage was then launched into the second OPA, consisting of a $1.4 \mathrm{~mm}$ long PPLN crystal. The second stage was pumped with $43 \mu \mathrm{J}$ and with beam waists corresponding to our experimental values of $186 \mu \mathrm{m}$ for the pump and $199 \mu \mathrm{m}$ for the seed. Through the calculation with

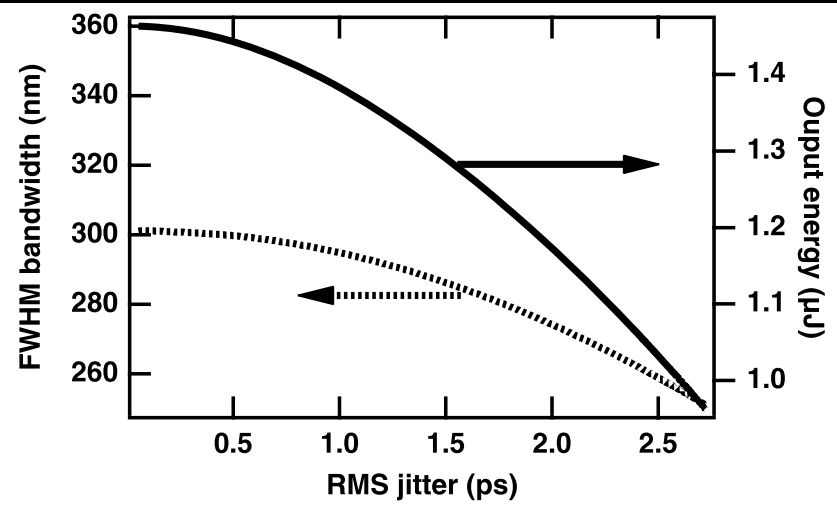

Fig. 10 Dependence of the output power from the two-stage OPCPA on timing jitter. The power is plotted for the optimum second OPA PPLN length of $1.4 \mathrm{~mm}$ and for a $2 \mathrm{~mm}$ long crystal. The OPA is pumped with $4.6 \mu \mathrm{J}$

a longer than optimum PPLN crystal, it is possible to determine the point inside the crystal where the maximum amplified seed energy is reached. The time delay between the seed and the pump is the same in the second stage as at the input to the first stage. For the simulations, we neglect additional timing jitter due to mechanical instabilities introduced after the splitting of the pump pulse for the two stages.

A normal distribution is assumed for the time delays introduced in our model and weighted averages of bandwidth and output energy are calculated for the different rms (root mean square) jitter values. Calculations have been performed with a resolution of $100 \mathrm{fs}$ and a temporal span of the delay ranging from -5 ps to 5 ps. Figure 10 depicts the results from our model. A timing jitter of 1 ps reduces the output energy by $5 \%$ whereas the bandwidth is only reduced by $2 \%$. Bandwidth is less affected than output energy. From the jitter simulations we can conclude that a timing jitter of less than $1 \mathrm{ps}$ rms should only have a minor impact on the performance of our OPCPA system. The PLL electronics of our experimental setup (model CLX-1100 from TimeBandwidth Products) measured an in-loop timing jitter of below 200 fs. This demonstrates that the repetition-rate stabilization by our electronics is sufficient for our application and no optical stabilization of the fiber and pump lasers are needed [49]. The latter would be difficult to implement with the combination of laser sources used in our setup.

\section{Alternative schemes}

The previous sections of this paper have clearly shown that the combination of a mode-locked picosecond solid-state and a femtosecond fiber laser amplifier system is a useful combination for efficient generation of broadband mid-IR pulses at high repetition rates, avoiding many of the drawbacks of the more conventional Ti:sapphire based technology. 
However, our simulations also show the bandwidth limitations of such an OPCPA system. At our operating wavelengths, the performance is limited by the phase-matching bandwidth of PPLN. Noncollinear phase-matching cannot solve this problem because of the low power level and narrow beams. Overall conversion efficiency has to be traded against bandwidth since the temporal profile of the pump implies weak stretching of the seed for broadband amplification. The resulting degradation of the temporal overlap with the pump pulse makes only limited use of the available pump energy. The best achievable conversion rate is $5 \%$ of the input power. This value is obtained by stretching the seed with $100 \mathrm{~mm}$ of sapphire and pumping the two stages at $3.9 \mu \mathrm{J}$ and $50 \mu \mathrm{J}$, respectively. This corresponds to a photon conversion efficiency of $19 \%$

One option to increase the efficiency is to use a flat top spatial beam profile instead of a Gaussian beam profile. To simulate the flat top beam we used a super-Gaussian of order 26 to pump the second OPA, while the first OPA was still pumped by a Gaussian beam profile. $50 \mathrm{~mm}$ of sapphire were used to stretch the seed. The flat beam profile increases the pulse energy after the second stage from $1.79 \mu \mathrm{J}$ to $2.36 \mu \mathrm{J}$ with a pump energy of $50 \mu \mathrm{J}$. Bandwidth slightly decreases from $335 \mathrm{~nm}$ to $320 \mathrm{~nm}$. A flat top temporal profile of the pump pulse would allow for longer stretching factors of the seed and thus for an improved temporal overlap with the pump.

\section{Conclusion}

We have shown the possibility to implement a femtosecond, high-repetition-rate, and high-power mid-IR OPCPA laser system starting from a commercially available diodepumped solid-state pump laser and fiber seed laser. Our numerical model predicts optimized OPCPA performance using two amplification stages. From the currently available mid-infrared gain materials we concluded that MgO:PPLN was the most suitable choice. The best performance of the system is obtained when stretching the seed pulses through $50 \mathrm{~mm}$ of sapphire material. Optimization of the two OPA stages made us choose $4 \mu \mathrm{J}$ of pump and a $3 \mathrm{~mm}$ long PPLN crystal for the first OPA. The remaining part of the pump is sent into the $1.4 \mathrm{~mm}$ long PPLN crystal of the second stage. Numerical simulations were used to determine the optimum beam sizes in both OPAs. The numerical model gave us detailed insight into the gain process and let us identify a mode of operation where some of the bandwidth limitations through gain narrowing could be overcome by operating the OPAs in strong saturation. Our two-stage OPCPA design amplifies the seed obtained through DFG with an energy of below $10 \mathrm{pJ}$ to the $\mu \mathrm{J}$-level. This corresponds to a gain of about $50 \mathrm{~dB}$ in a total of $4.4 \mathrm{~mm}$ PPLN material length. The OPCPA configuration optimized through simulations was built in our laboratory and the experimental results are consistent with the theoretical model [34]. To our knowledge, this is the first demonstration of an OPCPA system at such long wavelengths.

The proposed dual-stage OPCPA design is expected to become a promising experimental tool for many femtosecond mid-infrared physics and chemistry experiments. Such experiments greatly benefit from the high repetition rate of this system, leading to shorter measurement times and/or improved signal to noise ratios. The average power is significantly higher than most Ti:sapphire based optical parametric systems in this wavelength regime. Further scaling of repetition rate and average power seems feasible. For the future, a 50-W pump laser for further energy scaling and chirped quasi phase-matched gratings for improved amplification bandwidth will be investigated.

Acknowledgements We would like to thank Gunnar Arisholm for fruitful discussion and to acknowledge financial support by the NCCR Quantum Photonics (NCCR QP), research instrument of the Swiss National Science Foundation (SNSF) and Time-Bandwidth Products AG for their support with the DUETTO pump laser.

Open Access This article is distributed under the terms of the Creative Commons Attribution Noncommercial License which permits any noncommercial use, distribution, and reproduction in any medium, provided the original author(s) and source are credited.

\section{References}

1. S. Woutersen, U. Emmerichs, H.J. Bakker, Science 278, 658 (1997)

2. A.W. Omta, M.F. Kropman, S. Woutersen, H.J. Bakker, Science 301, 347 (2003)

3. M.L. Cowan, B.D. Bruner, N. Huse, J.R. Dwyer, B. Chugh, E.T.J. Nibbering, T. Elsaesser, R.J.D. Miller, Nature 434, 199 (2005)

4. J. Tate, T. Auguste, H.G. Muller, P. Salières, P. Agostini, L.F. DiMauro, Phys. Rev. Lett. 98, 013901 (2007)

5. A. Dubietis, G. Jonusauskas, A. Piskarskas, Opt. Commun. 88, 437 (1992)

6. F. Tauser, F. Adler, A. Leitenstorfer, Opt. Lett. 29, 516 (2004)

7. C. Erny, K. Moutzouris, J. Biegert, D. Kühlke, F. Adler, A. Leitenstorfer, U. Keller, Opt. Lett. 32, 1138 (2007)

8. D. Strickland, G. Mourou, Opt. Commun. 56, 219 (1985)

9. J. Rothhardt, S. Hädrich, D.N. Schimpf, J. Limpert, A. Tünnermann, Opt. Express 15, 16729 (2007)

10. A. Steinmann, A. Killi, G. Palmer, T. Binhammer, U. Morgner, Opt. Express 14, 10627 (2006)

11. M. Marangoni, R. Osellame, R. Ramponi, G. Cerullo, A. Steinmann, U. Morgner, Opt. Lett. 32, 1489 (2007)

12. M. Ghotbi, M. Ebrahim-Zadeh, V. Petrov, P. Tzankov, F. Noack, Opt. Express 14, 10621 (2006)

13. C.J. Fecko, J.J. Loparo, A. Tokmakoff, Opt. Commun. 241, 521 (2004)

14. J.-C. Wang, J.-K. Wang, J. Opt. Soc. Am. B 21, 45 (2004)

15. H. Maekawa, K. Tominaga, D. Podenas, Jpn. J. Appl. Phys. 41, $329(2002)$

16. T. Witte, D. Zeidler, D. Proch, K.L. Kompa, M. Motzkus, Opt. Lett. 27, 131 (2002) 
17. R.A. Kaindl, M. Wurm, K. Reimann, P. Hamm, A.M. Weiner, M. Woerner, J. Opt. Soc. Am. B 17, 2086 (2000)

18. F. Rotermund, V. Petrov, F. Noack, Opt. Quantum Electron. 32, 1057 (2000)

19. G.M. Gale, G. Gallot, F. Hache, R. Sander, Opt. Lett. 22, 1253 (1997)

20. U. Emmerichs, S. Woutersen, H.J. Bakker, J. Opt. Soc. Am. B 14, $1480(1997)$

21. V. Petrov, F. Noack, Opt. Lett. 21, 1576 (1996)

22. F. Seifert, V. Petrov, M. Woerner, Opt. Lett. 19, 2009 (1994)

23. B. Golubovic, M.K. Reed, Opt. Lett. 23, 1760 (1998)

24. J.A. Gruetzmacher, N.F. Scherer, Rev. Sci. Instrum. 73, 2227 (2002)

25. G.R. Holtom, R.A. Crowell, X.S. Xie, J. Opt. Soc. Am. B 12, 1723 (1995)

26. U. Emmerichs, H.J. Bakker, H. Kurz, Opt. Commun. 111, 497 (1994)

27. T. Fuji, N. Ishii, C.Y. Teisset, X. Gu, T. Metzger, A. Baltuška, N. Forget, D. Kaplan, A. Galvanauskas, F. Krausz, Opt. Lett. 31, 1103 (2006)

28. C.P. Hauri, R.B. Lopez-Matens, C.I. Blaga, K.D. Schultz, J. Cryan, R. Chirla, P. Colosimo, G. Doumy, A.M. March, C. Roedig, E. Sistrunk, J. Tate, J. Wheeler, L.F. DiMauro, Opt. Lett. 32, 868 (2007)

29. D. Brida, C. Manzoni, G. Cirmi, M. Marangoni, S.D. Silvestri, G. Cerullo, Opt. Express 15, 15035 (2007)

30. R. Laenen, K. Simeonidis, A. Lauberreau, J. Opt. Soc. Am. B 15, 1213 (1998)

31. F. Adler, K. Moutzouris, A. Leitenstorfer, H. Schnatz, B. Lipphardt, G. Grosche, F. Tauser, Opt. Express 12, 5872 (2004)

32. S.V. Marchese, C.R.E. Baer, A.G. Engqvist, S. Hashimoto, D.J.H.C. Maas, M. Golling, T. Südmeyer, U. Keller, Opt. Express 16, 6397 (2008)

33. J. Neuhaus, D. Bauer, J. Zhang, A. Killi, J. Kleinbauer, M. Kumkar, S. Weiler, M. Guina, D.H. Sutter, T. Dekorsy, Opt. Express 16, 20530 (2008)
34. C. Erny, C. Heese, M. Haag, L. Gallmann, U. Keller, Opt. Express 17, 1340 (2009)

35. O. Chalus, P.K. Bates, J. Biegert, Opt. Express 16, 21297 (2008)

36. G. Arisholm, Opt. Express 15, 6513 (2007)

37. L.E. Myers, R.C. Eckardt, M.M. Fejer, R.L. Byer, W.R. Bosenberg, Opt. Lett. 21, 591 (1996)

38. G. Cerullo, S.D. Silvestri, Rev. Sci. Instrum. 74, 1 (2003)

39. Y. Furukawa, K. Kitamura, S. Takekawa, K. Niwa, H. Hatano, Opt. Lett. 23, 1892 (1998)

40. Y. Furukawa, K. Kitamura, A. Alexandrovski, R.K. Route, M.M. Fejer, G. Foulon, Appl. Phys. Lett. 78, 1970 (2001)

41. U. Siegner, U. Keller, Nonlinear optical processes for ultrashort pulse generation, in Handbook of Optics, vol. III, ed. by M. Bass, E.W. Stryland, D.R. Williams, W.L. Wolfe (McGraw-Hill, New York, 2000)

42. G.M. Gale, F. Hache, M. Cavallari, IEEE J. Sel. Top. Quantum Electron. 4, 224 (1998)

43. V.G. Dmitriev, G.G. Gurzadyan, D.N. Nikogosyan, Handbook of Nonlinear Optical Crystals (Springer, Berlin, 1997)

44. T. Skauli, P.S. Kuo, K.L. Vodopyanov, T.J. Pinguet, O. Levi, L.A. Eyres, J.S. Harris, M.M. Fejer, B. Gerard, L. Becouarn, E. Lallier, J. Appl. Phys. 94, 6447 (2003)

45. G. Arisholm, J. Opt. Soc. Am. B 14, 2543 (1997)

46. C.P. Hauri, P. Schlup, G. Arisholm, J. Biegert, U. Keller, Opt. Lett. 29, 1369 (2004)

47. P. Schlup, J. Biegert, C.P. Hauri, G. Arisholm, U. Keller, Appl. Phys. B 79, 285 (2004)

48. M.J.W. Rodwell, D.M. Bloom, K.J. Weingarten, IEEE J. Quantum Electron. 25, 817 (1989)

49. N. Ishii, C.Y. Teisset, T. Fuji, S. Kohler, K. Schmid, L. Veisz, A. Baltuska, F. Krausz, IEEE J. Sel. Top. Quantum Electron. 12, 173 (2006) 